\title{
Suicídio em Idosos: índice e taxa de mortalidade nas capitais brasileiras no período de 2001 a 2015
}

\author{
Suicide in the Elderly: index and mortality rate in Brazilian capitals from 2001 to 2015 \\ John Victor dos Santos Silva ${ }^{1}$ (D) Claudio José dos Santos Júnior ${ }^{2}$ (D), \\ Keila Cristina Pereira do Nascimento Oliveira ${ }^{3}$ (D)
}

\section{RESUMO}

Fundamentos: o suicídio em idosos é um dos grandes problemas de saúde pública, que vem expressando taxas cada vez mais alarmantes pelo mundo atualmente. Objetivo: analisar o índice e taxa de mortalidade por suicídio em idosos nas capitais brasileiras no período de 2001 a 2015. Métodos: Trata-se de um estudo ecológico misto, com dados secundários coletados no Sistema de Indicadores de Saúde e Acompanhamento de Políticas Públicas do Idoso (SISAP IDOSO) e no Sistema de Informação sobre Mortalidade (SIM). A coleta ocorreu no período de setembro a novembro de 2018. Os dados foram tabulados e analisados descritivamente, com apresentação de gráficos e tabelas e frequências simples absolutas e percentuais. Principais resultados: $74,9 \%$ que cometeram suicídio foram homens e 53,7\% estavam entre 60 a 69 anos. A região sudeste apresentou maior índice de mortalidade, porém a maior taxa de mortalidade para cada 10.000 habitantes foi na região centro-oeste. Conclusão: há um crescente aumento nos índices e nas taxas de mortalidade de idosos, tornando-se uma preocupação para a saúde pública.

Palavras-chave: Suicídio; Idoso; Mortalidade; Saúde Pública.

\begin{abstract}
Background: Suicide in the elderly is one of the major public health problems, which has been expressing increasingly alarming rates worldwide today. Objective: Analyzing the suicide mortality rate and rate among the elderly in Brazilian capitals from 2001 to 2015. Methods: This is a mixed ecological study, with secondary data collected in the Sistema de Indicadores de Saúde e Acompanhamento de Políticas Públicas do Idoso (SISAP IDOSO) and Sistema de informação sobre Mortalidade (SIM). The collection was performed from September to November 2018. The data were tabulated and analyzed descriptively, with graphs, tables, and simple absolute and percentage frequencies. Main results: $74.9 \%$ who committed suicide were men, and $53.7 \%$ were between 60 and 69 years old. The southeastern region had the highest mortality rate, but the highest mortality rate for every 10,000 inhabitants was in the central-west region. Conclusion: there is a growing increase in the rates and mortality rates of the elderly, becoming a concern for public health.
\end{abstract}

Keywords: Suicide; Aged; Mortality; Public Health.

1. Programa de Pós-graduação em Enfermagem Psiquiátrica, Escola de Enfermagem de Ribeirão Preto, Universidade de São Paulo (EERP-USP), Ribeirão Preto (SP), Brasil.

2. Curso de Medicina, Centro de Ciências da Saúde, Universidade Estadual de Ciências da Saúde de Alagoas (UNCISAL), Maceió (AL), Brasil.

3. Escola de Enfermagem, Universidade Federal de Alagoas (UFAL), Maceió (AL), Brasil.

$\square$ John Victor dos Santos Silva. Avenida dos Bandeirantes, 3900, Campus Universitário - Bairro Monte Alegre. CEP: 14040-902. Ribeirão Preto (SP), Brasil.

john.setedejulho@gmail.com | Recebido em: 15/04/2020 | Aprovado em: 24/07/2020 


\section{INTRODUÇÃO}

O suicídio é um dos grandes problemas de saúde pública e que vem expressando taxas cada vez mais alarmantes pelo mundo ${ }^{1}$. Esse ato caracteriza-se por um fenômeno bastante complexo envolvendo inúmeros determinantes sociais, culturais, econômicos e de saúde, que leva o indivíduo à morte de forma planejada, provocada e intencional ${ }^{2}$.

A mortalidade por suicídio atinge cerca de 1 milhão de pessoas por ano no mundo e estima-se que em 2020 cerca de 1,5 milhão de pessoas cometerão suicídio ${ }^{3}$. No Brasil, apesar das taxas de mortalidade por suicídio serem baixas comparados aos demais países desenvolvidos e subdesenvolvidos do mundo, esses números têm crescido com o passar dos anos e têm se tornado preocupantes, principalmente entre os jovens e os idosos ${ }^{4}$.

O suicídio apresenta características que refletem em problemas sociais, demandando uma boa preparação dos serviços de saúde e de profissionais para atuar nessas circunstâncias 5 .

Os índices mais elevados de suicídio no mundo estão nos indivíduos do sexo masculino com 60 anos ou mais, em comparação as demais faixas etárias e, embora as taxas de suicídios no Brasil não sejam tão altas como em outros países desenvolvidos, a população que apresenta crescente manifestação e com maior risco para o suicídio são os idosos ${ }^{6}$.

Sabe-se que a população idosa no Brasil vem crescendo muito, resultado das mudanças econômicas, educacionais, e, principalmente, dos determinantes de saúde. Com isso, o suicídio vem crescendo nessa população, principalmente por conta das situações de vulnerabilidades, sejam elas socioeconômicas ou de saúde?

O envelhecimento é umas das fases do desenvolvimento humano com grandes mudanças biopsicossociais que podem trazer algumas limitações, perda de autonomia e importância no cunho social, além de algumas alterações e doenças características do processo de envelhecimento, o que pode levar a fatores que sejam considerados de risco para o comportamento suicida na população idosa ${ }^{8}$.

O suicídio, antes não muito investigado na população idosa, passa a ser preocupante, mobilizando profissionais de saúde para a identificação dos fatores de risco nesse grupo e também na criação de estratégias para trabalhar e propor para esses indivíduos um conjunto de ações de promoção da saúde e proteção da vida, favorecendo uma melhor percepção dos idosos sobre o significado da vida, ressignificando o sentido de viver ${ }^{9,10}$.

Assim, como na população em geral, os idosos do sexo masculino são os que mais cometem tentativa de suicídio com êxito chegando à morte. Identificar os fatores de risco para suicídio na população idosa é uma tarefa que necessita do trabalho conjunto dos familiares, comunidade e profissionais de saúde, possibilitando a identificação das vulnerabilidades que podem provocar nos idosos o desejo de descontinuidade da vida ${ }^{11}$.

Desta forma, o presente estudo teve como objetivo analisar o índice e a taxa de mortalidade por suicídio em idosos nas capitais brasileiras no período de 2001 a 2015.

\section{MATERIAIS E MÉTODOS}

Trata-se de um estudo ecológico misto realizado com dados secundários obtidos em sistema público e de livre acesso, dispensando a apreciação por parte do Comitê de Ética em Pesquisa da Instituição, em conformidade com a Resolução CNS/MS no 466/12.

\section{Participantes}

Foram incluídos no estudo os óbitos de indivíduos de 60 anos ou mais ocorridos entre o dia $1^{\circ}$ de janeiro de 2001 a 31 de dezembro de 2015 nas capitais dos 26 estados brasileiros e no Distrito Federal, coletados no Sistema de Indicadores de Saúde e Acompanhamento de Políticas Públicas do Idoso (SISAP IDOSO) através dos indicadores de mortalidade por suicídio; e também no Sistema de informação sobre Mortalidade (SIM) do Departamento de Informática do Sistema Único de Saúde (DATASUS), através dos óbitos decorrentes de lesões autoprovocadas intencionalmente.

\section{Material}

Os dados foram tabulados e armazenados no programa Excel, de acordo com a faixa etária, região geográfica e sexo do indivíduo, categorizadas a partir 
Classificação Estatística Internacional e Problemas Relacionados à Saúde - 10a Revisão (X60-84), e organizados de acordo com as faixas temporais, estabelecidas pelos quinquênios - 2001 a 2005, 2006 a 2010 e 2011 a 2015 - para a análise da mortalidade por suicídio em idoso por capital e por regiões geográficas. Ainda, foram calculadas as taxas de suicídio por 10.000 habitantes por sexo e relacionadas com as capitais brasileiras, onde os dados foram organizados de acordo com os anos 2001, 2006, 2010 e 2015.

\section{Procedimentos}

Foram realizadas análises exploratórias (descritivas) dos dados, a partir da apuração das frequências simples absolutas e percentuais para as variáveis. Realizou-se a descrição do perfil epidemiológico dos casos de tentativas de suicídio e óbitos por suicídio por meio de estatística descritiva e foram calculados os coeficientes de mortalidade ( 10.000 habitantes/ ano) por suicídio, e a taxa de habitantes foi retirada do Instituto Brasileiro de Geografia e Estatística (IBGE). Além disso, foi realizada a apresentação visual, empregando-se gráficos de linha da evolução dos óbitos por suicídio nos períodos: 2001, 2006, 2010 e 2015.

\section{RESULTADOS}

Houve, no período de 2001 a 2015, um total de 3.542 óbitos por suicídio em indivíduos com 60 anos ou mais nas capitais brasileiras, onde $74,9 \%$ (2.690) foram homens e $25,1 \%$ (852) foram mulheres. Com relação à faixa etária dos óbitos, verificou-se que $53,7 \%$ (1.903) foram na faixa etária de 60 e $69 ; 31,1 \%$ (1.101) na faixa de 70 e 79 ; e $15,2 \%$ (538) com 80 anos ou mais. As capitais da região sudeste concentram o maior número de óbito por suicídio com 48,2\% (1.712) do total, seguido das capitais das regiões nordeste com $21,8 \%$ (772), sul com $12,1 \%$ (429), centro-oeste com $11,8 \%$ (417), e por último a região norte com 6\% (212).

$\mathrm{O}$ índice geral de mortalidade em idosos por suicídio nas capitais brasileiras foi somado e apresentado por região geográfica, sexo, faixa etária e quinquênio, na Tabela 1.

Dentre as capitais brasileiras, São Paulo apresentou o maior índice de suicídio, concentrando
26,8\% (951) dos óbitos, sendo, também, de maior índice entre as capitais do Sudeste, apresentando $55,5 \%$ (951). No Sul do país, Florianópolis apresentou o maior índice entre as capitais com 55,5\% (238) óbitos. Na região Nordeste, a capital que apresentou maior índice foi Fortaleza com 30,7\% (237); na região Norte o maior índice apresentado foi de 33,5\% (71) em Manaus, no Amazonas.

Dos 3542 óbitos registrados entre os idosos nas capitais brasileiras entre os anos de 2001 e 2015, verificou-se que $28,6 \%$ (1.014) aconteceram no quinquênio de $2001-2005 ; 31,3 \%$ (1.108) no período 2006-2010, com crescimento de 9,3\% em comparação ao quinquênio anterior; e 40,1\% (1.420) no quinquênio 2011-2015, com crescimento de $28,2 \%$ em comparação ao quinquênio anterior. $O$ maior índice de óbitos por suicídio em idosos, somando homens e mulheres, concentra-se na faixa etária de 60 e 69 anos (Tabela 2).

Quando verificada a taxa de mortalidade de idosos por suicídio para cada 10.000 habitantes, contatou-se que os homens apresentam a maior taxa de mortalidade, tendo significativa variação ao longo dos quinquênios, enquanto as mulheres permaneceram com taxa constante ao longo do período, apresentando um apequena variação apenas no ano de 2015. No período de 2006 e 2011, a taxa geral de mortalidade de idosos por suicídio para cada 10.000 habitantes foi a mesma, como mostra a Tabela 3.

Comparou-se o número geral de suicídio em idosos nas capitais por regiões brasileiras com as taxas de mortalidade em idosos por suicídio para cada 10.000 habitantes, notando diferença significativa nas capitais das regiões que apresentam maior e menor índice de mortalidade por suicídio em idosos.

Como podemos ver na Figura 1, em 2001, as capitais da região Norte apresentaram a maior taxa de suicídios entre idosos por 10.000 habitantes. Em 2006 houve uma grande diminuição nesses valores, e as capitais da região Centro-Oeste insurgiram com maior número suicídios no período. Em 2011, as capitais da região Centro-Oeste continuaram sendo a região com o maior número de suicídios de idosos e com uma taxa maior que as dos anos anteriores. No ano de 2015 houve uma queda acentuada no número de suicídios nas capitais da região Centro-Oeste, e a taxa por 10 mil habitantes, sendo a mesma das capitais das regiões Nordeste e Norte, ficando a região Sul com a maior taxa de mortalidade. 
Tabela 1

Distribuição dos óbitos por suicídio nas capitais, somados e apresentados por região geográfica, sexo, faixa etária e quinquênio.

\begin{tabular}{|c|c|c|c|c|c|c|c|c|}
\hline \multirow{2}{*}{ Região Geográfica } & \multirow{2}{*}{ Sexo } & \multirow{2}{*}{ Faixa etária } & \multicolumn{2}{|c|}{ 2001-2005 } & \multicolumn{2}{|c|}{ 2006-2010 } & \multicolumn{2}{|c|}{ 2011-2015 } \\
\hline & & & $\mathbf{n}$ & $\%$ & $\mathbf{n}$ & $\%$ & $\mathbf{n}$ & $\%$ \\
\hline \multirow{6}{*}{ Norte } & & $60-69$ & 7 & 64 & 3 & 43 & 10 & 63 \\
\hline & Mulher & $70-79$ & 2 & 18 & 3 & 43 & 3 & 19 \\
\hline & & 80 ou + & 2 & 18 & 1 & 14 & 3 & 19 \\
\hline & & $60-69$ & 25 & 51 & 21 & 41 & 50 & 64 \\
\hline & Homem & $70-79$ & 20 & 41 & 25 & 49 & 23 & 29 \\
\hline & & 80 ou + & 4 & 8 & 5 & 10 & 5 & 6 \\
\hline \multirow{6}{*}{ Nordeste } & & $60-69$ & 20 & 51 & 28 & 61 & 54 & 58 \\
\hline & Mulher & $70-79$ & 17 & 44 & 15 & 33 & 31 & 33 \\
\hline & & 80 ou + & 2 & 5 & 3 & 7 & 8 & 9 \\
\hline & & $60-69$ & 89 & 54 & 98 & 53 & 134 & 55 \\
\hline & Homem & $70-79$ & 55 & 34 & 63 & 34 & 76 & 31 \\
\hline & & 80 ou + & 20 & 12 & 25 & 13 & 34 & 14 \\
\hline \multirow{6}{*}{ Centro-Oeste } & & $60-69$ & 13 & 62 & 14 & 61 & 16 & 44 \\
\hline & Mulher & $70-79$ & 5 & 24 & 4 & 17 & 13 & 36 \\
\hline & & 80 ou + & 3 & 14 & 5 & 22 & 7 & 19 \\
\hline & & $60-69$ & 52 & 54 & 61 & 56 & 53 & 42 \\
\hline & Homem & $70-79$ & 28 & 29 & 28 & 26 & 41 & 33 \\
\hline & & 80 ou + & 17 & 18 & 19 & 18 & 31 & 25 \\
\hline \multirow{6}{*}{ Sudeste } & & $60-69$ & 68 & 51 & 86 & 57 & 110 & 59 \\
\hline & Mulher & $70-79$ & 51 & 38 & 40 & 26 & 50 & 27 \\
\hline & & 80 ou + & 15 & 11 & 25 & 17 & 27 & 14 \\
\hline & & $60-69$ & 201 & 54 & 231 & 57 & 229 & 49 \\
\hline & Homem & $70-79$ & 115 & 31 & 110 & 27 & 147 & 32 \\
\hline & & 80 ou + & 57 & 15 & 67 & 16 & 87 & 19 \\
\hline \multirow{6}{*}{ Sul } & & $60-69$ & 12 & 52 & 20 & 69 & 33 & 77 \\
\hline & Mulher & $70-79$ & 10 & 43 & 5 & 17 & 7 & 16 \\
\hline & & 80 ou + & 1 & 4 & 4 & 14 & 3 & 7 \\
\hline & & $60-69$ & 47 & 46 & 48 & 48 & 70 & 52 \\
\hline & Homem & 70-79 & 41 & 40 & 31 & 31 & 42 & 31 \\
\hline & & 80 ou + & 15 & 15 & 20 & 20 & 23 & 17 \\
\hline
\end{tabular}

FONTE: SISAP IDOSO; DATASUS/SIM, 2018.

Tabela 2

Óbitos por suicídio nas capitais por faixa etária e quinquênio, 2019.

\begin{tabular}{|c|c|c|c|c|c|c|}
\hline \multirow{2}{*}{ Faixa etária } & \multicolumn{2}{|c|}{ 2001-2005 } & \multicolumn{2}{|c|}{$2006-2010$} & \multicolumn{2}{|c|}{ 2011-2015 } \\
\hline & $\mathbf{n}$ & $\%$ & $\mathbf{n}$ & $\%$ & $\mathbf{N}$ & $\%$ \\
\hline 60-69 anos & 534 & 53 & 610 & 55 & 759 & 53 \\
\hline 70-79 anos & 344 & 34 & 324 & 29 & 433 & 31 \\
\hline 80 ou + & 136 & 13 & 174 & 16 & 228 & 16 \\
\hline
\end{tabular}

FONTE: SISAP IDOSO; DATASUS / SIM, 2018. 
Tabela 3

Taxa de suicídios em idosos para cada 10.000 habitantes segundo sexo entre os anos de 2001, 2006, 2010 e 2015.

\begin{tabular}{lcccc}
\hline & $\mathbf{2 0 0 1}$ & $\mathbf{2 0 0 6}$ & $\mathbf{2 0 1 1}$ & $\mathbf{2 0 1 5}$ \\
\hline Homem & 0.99 & 1.06 & 0.97 & 0.98 \\
Mulher & 0.20 & 0.20 & 0.20 & 0.23 \\
Todos & 0.57 & 0.54 & 0.54 & 0.55 \\
\hline
\end{tabular}

FONTE: SISAP IDOSO; DATASUS/SIM, 2018.

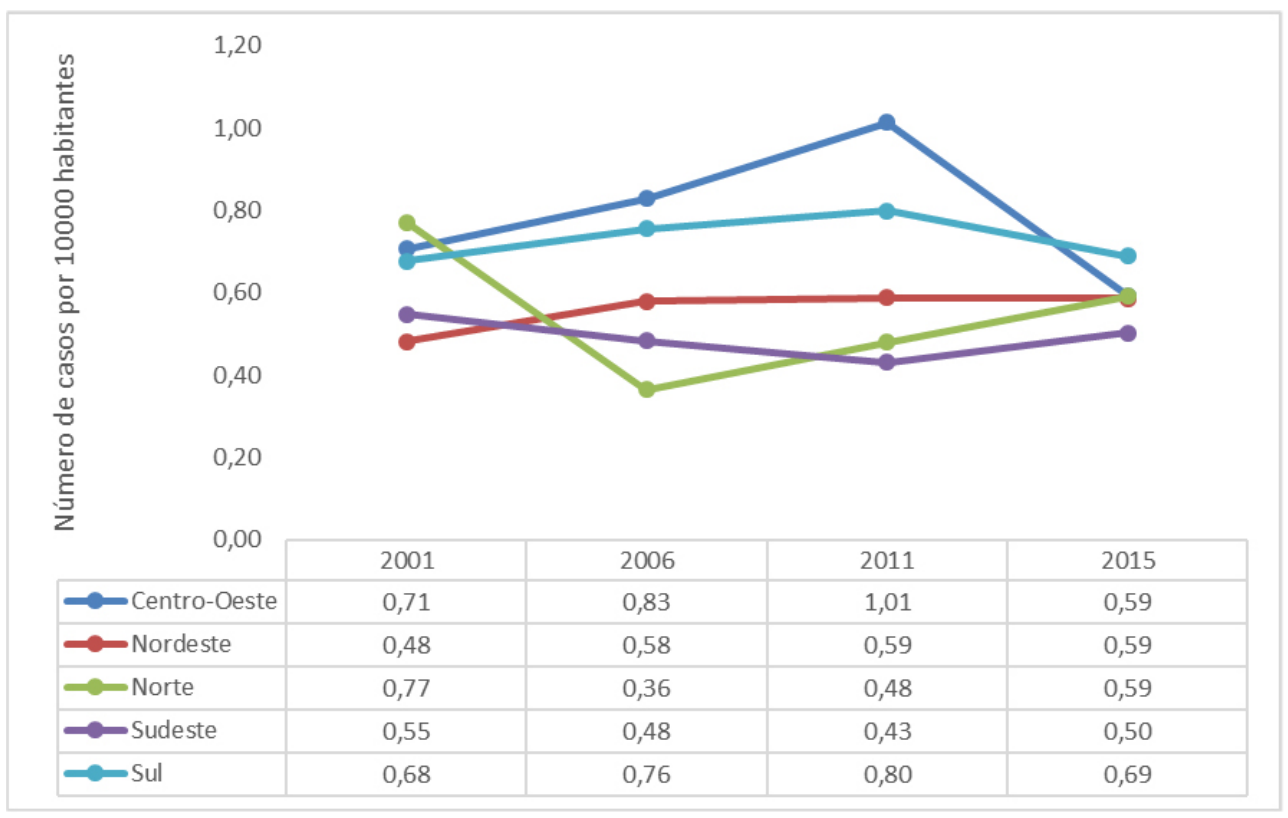

Figura 1: Taxa de suicídios para cada 10.000 habitantes das capitais por regiões geográficas, entre os anos 2001, 2006, 2010 e 2015 .

FONTE: SISAP IDOSO; DATASUS/SIM, 2018.

Na região Norte, Palmas apresentou a maior taxa de mortalidade e Manaus a menor, em 2001; Boa Vista e Porto Velho tiveram ambas as maiores taxas e Macapá e Palmas as menores, em 2016; Boa vista continuou com a maior taxa em 2011 e Belém apresentou a menor; em 2015, Macapá apresentou a maior taxa e Belém permaneceu com a menor taxa.

Na Região Nordeste, a capital com a maior taxa de mortalidade em 2001 foi Recife e a menor foi Salvador. Em 2006, quem apresentou a maior taxa foi Fortaleza e a menor foi Natal. Em 2011, quem apresentou a maior taxa foi Teresina e as menores taxas foram Recife e Natal. Já em 2015, São Luís apresentou a maior taxa de mortalidade e a menor foi Natal.
Na região Centro-Oeste, em 2001, a capital que apresentou maior taxa de mortalidade por suicídio em idosos foi Goiânia e a menor foi Cuiabá. No ano de 2006 quem apresentou maior taxa foi Campo Grande e a menor foi em Goiânia, que em 2011 e 2015 voltou a ter a maior taxa da região e Cuiabá com a menor taxa da região nos dois períodos.

Na região Sudeste, São Paulo apresentou as maiores taxas da região em 2001 e 2006, e as menores foram Belo Horizonte e Vitória, consecutivamente. Em 2011, Belo Horizonte passou a apresentar a maior taxa de mortalidade e Vitória continuou com a menor taxa, porém em 2015, Vitória passou a ter a maior taxa de mortalidade e o Rio de Janeiro, a menor. 
$\mathrm{Na}$ região sul, Florianópolis apresentou a maior taxa de mortalidade em 2001 e Porto Alegre a menor. Florianópolis passou a apresentar a menor taxa da região em 2006, Curitiba a menor em 2011 e Florianópolis a menor em 2015. Porto Alegre apresentou maiores taxas em 2006, 2011 e 2015.

\section{DISCUSSÃO}

Existe uma concordância entre os dados da Organização Mundial de Saúde e os diversos estudos existentes sobre a prática do suicídio no mundo, onde o público que mais comete suicídio é do

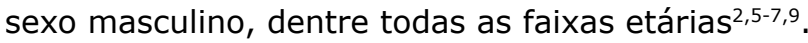
Além disso, Santos et al. ${ }^{11}$ mostraram que essa taxa se dá pelas formas mais articuladas, planejadas, pesadas e eficazes utilizadas pelos homens, sendo meios que nas maiorias das vezes não há chances de sobrevida, diferentemente das estratégias utilizadas pelas mulheres.

Minayo, Meneghel e Cavalcante ${ }^{12}$ mostram que uma das principais causas de morte por suicídio entre os idosos do sexo masculino é relacionada à hegemonia da masculinidade, ainda muito expressiva na sociedade atualmente. Quando o homem tem as suas funções sociais consideradas masculinas afetadas, principalmente pela dependência, seja ela financeira, emocional, de saúde entre outras, isso se torna um grande fator de risco.

Levando em conta que historicamente o homem sempre foi considerado a figura de poder, de liderança, de chefia da família, do trabalho e das relações sociais, quando essa figura é abalada de alguma forma, o homem se considera impotente, aumentando a possibilidade de isolamento social e risco para problemas emocionais?

Já um estudo realizado por Silva et al. ${ }^{13} \mathrm{em}$ algumas cidades da região Nordeste mostrou que o risco de suicídio entre as idosas é maior quando as mesmas possuem baixa escolaridade, dependência de familiares e principalmente do cônjuge, além de sofrerem algum tipo de violência doméstica, seja sexual, física ou psicológica.

Meneghel et al. ${ }^{14}$ trazem ainda que as mulheres procuram estratégias mais brandas para $o$ ato suicida, isso revela o porquê as mulheres tentam mais o suicídio, pois os meios utilizados apresentam chances de sobrevida quando a assistência é imediata, favorecendo, quando não prestada uma atenção acompanhada, a predisposição para que as idosas cometam mais uma vez a tentativa, mostrando assim que a mortalidade entre os homens é maior, enquanto entre as mulheres a tentativa é mais frequente.

Quanto à faixa etária dos idosos que cometem suicídio, um estudo realizado na Bahia por Carmo et al. ${ }^{15}$ corroborou com os dados desta pesquisa, mostrando que o maior número de óbitos em idosos por suicídio está entre 60 e 69 anos, acontecendo em $53,8 \%$ dos casos, similar a este estudo.

Um estudo realizado em outro estado do nordeste do país, no Instituto Médico Legal do Piauí, mostrou que entre o período de 2007 e 2014 aconteceram 79 óbitos de idosos por tentativa de suicídio em âmbito hospitalar, onde $54 \%$ foram na faixa etária entre 60 e 70 anos, corroborando mais uma vez com o presente estudo ${ }^{8}$.

D'eça Jr et al. ${ }^{16}$ mostraram, em um estudo realizado sobre o suicídio no Brasil no período de 1996 até 2015, que o maior índice de suicídio no país concentra-se na região Sudoeste, seguindo da região Sul, Nordeste, Centro-Oeste e o menor índice no Norte, porém esse estudo é uma tendência geral dos suicídios do país, enquanto o presente é um estudo apenas nas capitais e com os idosos, mostrando que a ordem dos índices nas capitais é Sudeste, Nordeste, Sul, Centro-Oeste e Norte.

Pinto e Assis ${ }^{17}$ trazem em seu estudo que na região Sudeste a maior taxa de óbitos de idosos por suicídio é no estado de Minas Gerais. Percebe-se grande diferença quando avaliado o índice de suicídio no estado e nas capitais.

Esperava-se que nas grandes metrópoles dos estados e suas capitais a tendência de suicídio dos idosos fosse proporcional nas regiões, porém quando avaliados separadamente, podemos perceber diferença nas taxas por capital, por estado e por regiões brasileiras, não seguindo um padrão, porém com diferenças significativas da distribuição espacial ${ }^{6,4,7,15-18}$.

A pesquisa realizada por Santos et al. ${ }^{19}$ mostra um crescente aumento no número de óbitos de idosos por suicídio no período de 2000 até 2014 , onde o maior número de casos aconteceu no quinquênio de 2010 e 2014, corroborando com o presente estudo que mostrou um crescente aumento no índice, sendo o maior número de óbitos entre 2011 e 2015.

A taxa de mortalidade entre os homens idosos é maior do que entre as mulheres idosas ${ }^{3}$. Alguns 
estudos mostram estabilidade na taxa de mortalidade de idosos do sexo masculino comparado ao crescimento da taxa de mortalidade dos idosos do sexo masculinos no Brasil ${ }^{5,8,15}$, porém Pinto e Assis ${ }^{17}$ mostram que há um crescimento significativo nos óbitos de idosos do sexo feminino, por causa das condições socioeconômicas e regionais.

Embora o presente estudo tenha trazido que as capitais da região Centro-Oeste do Brasil apresentam a maior taxa de óbito de idosos por suicídio, outros estudos realizados mostram que quando verificada toda a região (não apenas as capitais), a região do Brasil que apresenta maior taxa de suicídio no geral é a região Sul, seguida do Nordeste, levando em consideração a taxa de mortalidade para cada 100.000 habitantes $5,7,9,15,16$.

Deve-se levar em consideração que as regiões brasileiras possuem diferenças geográficas, climáticas, econômicas, educacionais, entre outras, e todas essas questões pesam no momento da avaliação do índice de suicídio ${ }^{6}$. Os fatores emocionais são importantes na hora de identificar o ato suicida, pois mais de $80 \%$ dos casos estão relacionados a um grande sofrimento mental enfrentado principalmente em questões de saúde, diante de um diagnóstico de uma doença crônica ou incurável ${ }^{20}$.

A maioria dos estudos apresenta sempre a região Norte com a menor taxa e índice de suicídio em geral ${ }^{5,8,9}$, porém Pinto e Assis ${ }^{17}$ mostraram um crescimento nas taxas de óbitos de idosos por suicídio. Na região Nordeste, Silva et al. ${ }^{13}$ mostraram que a região do Nordeste, principalmente no estado do Ceará, apresentou grandes índices de tentativas de suicídio, principalmente entre as idosas. Em Alagoas esses índices são mais discretos, porém preocupantes, do ponto de vista da subnotificação ${ }^{18}$.

A região Centro-Oeste é apontada como uma das que concentram maiores taxas de suicídios entre os idosos, como mostra a pesquisa realizada por Santos et al. ${ }^{19}$ e D'eça Jr et al. ${ }^{16}$ mostrando as altas taxas de mortalidade na população em geral e na população idosa. Uma pesquisa feita por Cabral e Pendloski ${ }^{3}$ no sul do país mostrou que o estado que apresenta maior taxa de mortalidade de idosos por suicídio foi Rio Grande do Sul, o que mostra uma discreta relação com os resultados desta pesquisa.

Todo esse crescimento do índice de suicídios entre os idosos mostra-se preocupante para os serviços de saúde, necessitando maior conhecimento sobre os motivos que realmente levam os idosos a desejarem colocar fim a suas vidas e no desenvolvimento de estratégias que possam ajudar a diminuir essa taxa, favorecendo a promoção e proteção da vida, pois é importante entender que é um esforço coletivo ${ }^{21}$.

\section{CONCLUSÃO}

De acordo com o objetivo e resultados desse estudo conclui-se que $74,9 \%$ dos idosos que cometeram suicídio nas capitais brasileiras foram do sexo masculino e $53,7 \%$ com faixa etária de 60 a 69 anos. A região brasileira que apresentou a maior mortalidade em suas capitais foi a região sudeste, enquanto que a menor foi a região Norte. Constatou-se também que o maior número de mortes foi no quinquênio de 2011-2015.

Foi analisada a taxa de mortalidade para cada 10.000 habitantes e verificou-se que os idosos detiveram a maior taxa de mortalidade do que as idosas, no qual as mesmas mantiveram uma taxa linear nos dois primeiros quinquênios apresentando apenas um leve aumento no último quinquênio. As capitais da região centro-oeste apresentou maior mortalidade para cada 10.000 habitantes, com a maior taxa de mortalidade de idosos por suicídio entre as demais capitais brasileiras.

Embora todas as diferenças significantes nas taxas de suicídio entre as capitais, estados e regiões brasileiras, existe um consenso de que os índices aumentam com o passar dos anos, se tornando cada vez mais preocupantes para os idosos, sendo necessários novos estudos para conhecerem as reais causas e também estratégias eficazes que possam diminuir a esses indicies e ações que promovam a qualidade e proteção da vida.

\section{CONFLITO DE INTERESSES}

Não há.

\section{FONTE DE FOMENTO}

Conselho Nacional de Desenvolvimento Científico e Tecnológico (CNPq). 


\section{APROVAÇÃO DO COMITÊ DE ÉTICA EM PESQUISA}

Não se aplica.

\section{REGISTRO DOS ENSAIOS CLÍNICOS EM BASE DE ACESSO PÚBLICO}

Não se aplica.

\section{REFERÊNCIAS}

1. World Health Organization. World report on violence and health [Internet]. Geneve; 2002. Disponível em: https://apps.who.int/iris/bitstream/handle/10665 /42495/9241545615_eng.pdf; jsessionid=2AE28B4F7D6F5ABB386CC95518E49B72? sequence $=1$. Acesso em: 15 de Abril de 2020.

2. World Health Organization. Preventing suicide: a global imperative [Internet]. Geneva 2014. Disponível em: https://www.who.int/mental_health/suicide-prevention/ world_report_2014/en/. Acesso em: 15 de abril de 2020.

3. Cabral DVS, Pendloski J. Mortalidade por suicídio em idosos: uma análise do perfil epidemiológico no sul do brasil. REVISTA UNINGÁ, 2016;47(2):19-24.

4. Bahia CA, Avanci JQ, Pinto LW, Minayo MCDS. Lesão autoprovocada em todos os ciclos da vida: perfil das vítimas em serviços de urgência e emergência de capitais do Brasil. Ciência \& Saúde Coletiva, 2017;22(9):2841-50. DOI: $10.1590 / 1413-81232017229.12242017$.

5. Calixto Filho M, Zerbini T. Epidemiologia do suicídio no Brasil entre os anos de 2000 e 2010. Saúde, Ética \& Justiça, 2016;21(2):45-51. DOI: 10.11606/issn.23172770.v21i2p45-51.

6. Botega NJ. Comportamento suicida: epidemiologia. Psicologia Usp, 2015;25(3):231-236. DOI: 10.1590/ 0103-6564D20140004.

7. Santos RS, Albuquerque MCD, Brêda MZ, Bastos MLDA, Silva VMS, Tavares NVDS. A atuação do enfermeiro com a pessoa em situação de suicídio: análise reflexiva. Rev. enferm. UFPE, 2017;11(2):742-748.

8. Gomes AV, Cardoso PKB, Rocha FCV, Carvalho CMSD, Sales MCV. Perfil sociodemográfico de idosos vítimas de suicídio em um estado do Nordeste do Brasil. Rev. baiana enferm, 2018;32:e26078. DOI: 10.18471/rbe.v32.26078.

9. Pinto AP, Carvalho ML, Amorim LMM, Linhares RC, Lima FF et al. SUICÍDIO EM IDOSOS NO BRASIL: uma revisão integrativa. Revista Univap, 2016;22(40):849.

10. Almeida BLS, Lorentz M, Bertoldo LTM. Aspectos Psicossociais do Suicídio em Idosos e Percepções de Sobreviventes. Revista de Psicologia da IMED, 2018;10(1):21-36. DOI: 10.18256/2175-5027.2018. v10i1.2260.

11. Santos EDGM, Lira GOL, Santos LM, Alves MÊS, Araújo LF et al. Suicídio entre idosos no Brasil: uma revisão de literatura dos últimos 10 anos. Psicología, Conocimiento y Sociedad, 2019;9(1):258-282. DOI: 10.26864/PCS. v9.n1.12.

12. Minayo MCS, Meneghel SN, Cavalcante FG. Suicídio de homens idosos no Brasil. Ciência \& Saúde Coletiva, 2012;17(10):2665-74. DOI: 10.1590/ S1413-81232012001000016.

13. Silva RMD, Sousa GSD, Vieira LJEDS, Caldas JMP, Minayo MCS. Ideação e tentativa de suicídio de mulheres idosas no nordeste do Brasil. Revista Brasileira de Enfermagem, 2018;71(2):755-62. DOI: 10.1590/0034-7167-2017-0413.

14. Meneghel SN, Moura R, Hesler LZ, Gutierrez DMD. Tentativa de suicídio em mulheresidosas-uma perspectiva de gênero. Ciência \& Saúde Coletiva, 2015;20(6):172130. DOI: $10.1590 / 1413-81232015206.02112015$.

15. Carmo EA, Santos PHS, Ribeiro BS, Soares CJ, Santana MLAD et al. Características sociodemográficas e série temporal da mortalidade por suicídio em idosos no estado da Bahia, 1996-2013. Epidemiologia e Serviços de Saúde, 2018;27(1):e20171971. DOI: 10.5123/ s1679-49742018000100001

16. D'Eça Júnior $A$, Rodrigues LDS, Meneses Filho EP, Costa LDLN, Rêgo ADS et al. Mortality by suicide in the Brazilian population, 1996-2015: what's the predominant trend?. Cadernos Saúde Coletiva, 2019;27(1):2024. DOI: $10.1590 / 1414-462 \times 201900010211$.

17. Pinto LW, Assis SGD. Estudo descritivo das tentativas de suicídio na população idosa brasileira, 20002014. Ciência \& Saúde Coletiva, 2015;20(6):1681-92. DOI: $10.1590 / 1413-81232015206.03532015$.

18. Santos Júnior CJ, Santos IV, Silva JVS, Gomes VM, Ribeiro MC. Perfil de pacientes atendidos por tentativa de suicídio em um Hospital Geral de Emergências do estado de Alagoas, Brasil. Medicina (Ribeirao Preto Online), 2019;52(3):223-30. DOI: 10.11606/issn.21767262.v52i3.p223-230.

19. Santos EGO, Oliveira YOMCO, Azevedo UM, Nunes ADS, Amador AE, Barbosa IR. Análise espaço-temporal da mortalidade por suicídio em idosos no Brasil. Rev Bras Geriatr. Gerontol. 2017;20(6):854-65. DOI: 10.1590/1981-22562017020.170115.

20. Santos MA. Câncer e suicídio em idosos: determinantes psicossociais do risco, psicopatologia e oportunidades para prevenção. Ciência \& Saúde Coletiva, 2017;22(9):3061-75. DOI: $10.1590 / 1413-81232017229.05882016$.

21. Machado DB, Santos DND. Suicide in Brazil, from 2000 to 2012. Jornal Brasileiro de Psiquiatria, 2015;64(1):4554. DOI: $10.1590 / 0047-2085000000056$. 